\title{
INTEGRAL, XMM-Newton and ESO/NTT identification of AX J1749.1-2733: an obscured and probably distant Be/X-ray binary ${ }^{\star}$
}

\author{
J. A. Zurita Heras and S. Chaty
}

\author{
Laboratoire AIM, CEA/DSM-CNRS-Université Paris Diderot, IRFU/Service d'Astrophysique, Bât. 709, CEA-Saclay, \\ 91191 Gif-sur-Yvette, France \\ e-mail: [juan-antonio.zurita-heras; sylvain.chaty]@cea.fr
}

Received 19 November 2007 / Accepted 18 June 2008

\begin{abstract}
Context. AX J1749.1-2733 is an unclassified transient X-ray source discovered during surveys by ASCA in 1993-1999. The transient behaviour and the short and bright flares of the source have led to the idea that it is part of the recently revealed subclass of supergiant fast X-ray transients.

Aims. A multi-wavelength study in NIR, optical, X-rays, and hard X-rays of AX J1749.1-2733 is undertaken in order to determine its nature.

Methods. Public INTEGRAL data and our target of opportunity observation with XMM-Newton were used to study the high-energy source through timing and spectral analysis. Multi-wavelength observations in optical and NIR with the ESO/NTT telescope were also performed to search for the counterpart.

Results. AX J1749.1-2733 is a new high-mass X-ray binary pulsar with an orbital period of $185.5 \pm 1.1$ days (or $185.5 / f$ with $f=2,3$ or 4 ) and a spin period of $\sim 66 \mathrm{~s}$, parameters typical of a Be/X-ray binary. The outbursts last $\sim 12 \mathrm{~d}$. A spin-down of $\dot{P}=0.08 \pm$ $0.02 \mathrm{~s} \mathrm{yr}^{-1}$ is also observed, very likely due to the propeller effect. The most accurate X-ray position is RA $(2000)=17^{\mathrm{h}} 49^{\mathrm{m}} 06.8^{\mathrm{s}}$ and Dec $=-27^{\circ} 32^{\prime} 32.5^{\prime \prime}$ (uncertainty $2^{\prime \prime}$ ). The high-energy broad-band spectrum is well-fitted with an absorbed powerlaw and a highenergy cutoff with values $N_{\mathrm{H}}=20.1_{-1.3}^{+1.5} \times 10^{22} \mathrm{~cm}^{-2}, \Gamma=1.0_{-0.3}^{+0.1}$, and $E_{\text {cut }}=21_{-3}^{+5} \mathrm{keV}$. The only optical/NIR candidate counterpart within the X-ray error circle has magnitudes of $R=21.9 \pm 0.1, I=20.92 \pm 0.09, J=17.42 \pm 0.03, H=16.71 \pm 0.02$, and $K_{\mathrm{S}}=15.75 \pm 0.07$, which points towards a Be star located far away $(>8.5 \mathrm{kpc})$ and highly absorbed $\left(N_{\mathrm{H}} \sim 1.7 \times 10^{22} \mathrm{~cm}^{-2}\right)$. The average 22-50 keV luminosity is $0.4-0.9 \times 10^{36} \mathrm{erg} \mathrm{s}^{-1}$ during the long outbursts and $3 \times 10^{36} \mathrm{erg} \mathrm{s}^{-1}$ during the bright flare that occurred on MJD 52891 for an assumed distance of $8.5 \mathrm{kpc}$.
\end{abstract}

Key words. X-ray: binaries - X-rays: individuals: AX J1749.1-2733 - infrared: stars

\section{Introduction}

High-mass X-ray binaries (HMXB) are systems composed of a compact object (either a neutron star (NS) or a black hole $(\mathrm{BH})$ ) and a massive companion star. The emission in X-rays is explained by the accretion of the stellar wind coming from the companion and captured by the compact object. There are mainly two classes of HMXB, depending on the nature of the massive primary star, which has important implications for the X-ray observational features. One might read White et al. (1995) and Psaltis (2006, and references therein) for reviews of HMXB.

The first class is composed of persistent high-energy binary systems made of a supergiant star (spectral type OB) (later called SGXB) orbiting close to the compact object. The other group is composed of a Be star accompanying the compact object, mostly an NS. A Be star is a fast-rotating B star possessing a dense and

\footnotetext{
* Based on observations made with 1) INTEGRAL, an ESA project with instruments and science data centre funded by ESA member states (especially the PI countries: Denmark, France, Germany, Italy, Switzerland, Spain), Czech Republic and Poland, and with the participation of Russia and the USA; 2) XMM-Newton, an ESA science mission with instruments and contributions directly funded by ESA Member States and NASA; and 3) ESO Telescopes at the La Silla or Paranal Observatories under programme ID 079.D-0432(A).
}

slow stellar outflow at the equator (thus creating a disc-like ring of matter surrounding the $\mathrm{B}$ star). The group of $\mathrm{BeXB}$ is the most numerous in the HMXB family (Liu et al. 2000, 2006). They possess wide orbits ( $P_{\text {orb }}>15$ days). They display either recurrent outbursts near the periastron passage whose duration is normally linked to the orbital period (most common outbursts), or giant outbursts that can last from weeks to months. In most cases, their X-ray emission is modulated by the strong magnetic field of the NS. Their spectra are similar to those of supergiant X-ray binaries (see reviews of Bildsten et al. 1997; Coe 2000; Negueruela 2007).

Among the new sources, INTEGRAL might have unveiled a new subclass of SGXB. Indeed, several newly discovered sources were identified as galactic X-ray sources with a supergiant companion, and they display transient behaviour (e.g. IGR J17544-2619, in't Zand 2005; Pellizza et al. 2006). The quiescent level in these systems is of the order of $10^{32-33} \mathrm{erg} \mathrm{s}^{-1}$ (e.g. IGR J08408-4503, Götz et al. 2007; Leyder et al. 2007). The X-ray luminosity increases up to $10^{36} \mathrm{erg} \mathrm{s}^{-1}$ (as observed in the known SGXB) only during short luminous-flare activity. The X-ray luminosity remains at a very low level (if not totally absent) during most of the time, except during the flares. These flaring periods last for a few hours at most, and then the source goes back to an undetectable level of emission (e.g. 
XTE J1739-302, Smith et al. 2006). Therefore, they received the name of Supergiant Fast X-ray Transient (SFXT) (Negueruela et al. 2006). Except for the transient nature of SFXT, their spectral features are similar to the previously known persistent SGXB.

The unclassified transient X-ray source AX J1749.1-2733 was discovered at a very low-luminosity level during the ASCA galactic centre survey performed between 1993 and 1999 (Sakano et al. 2002). Its position is RA (2000) = $17^{\mathrm{h}} 49^{\mathrm{m}} 09.0^{\mathrm{s}}$ and $\mathrm{Dec}=-27^{\circ} 33^{\prime} 13.9^{\prime \prime}\left(50^{\prime \prime}\right.$ at $90 \%$ confidence). The source was detected three times over the six observations when the source was located within the instrument field of view. The observed $0.7-10 \mathrm{keV}$ flux varies between $(2-6) \times 10^{-12} \mathrm{erg} \mathrm{cm}^{-2} \mathrm{~s}^{-1}$. Each spectrum is fitted with an absorbed power-law model. However, only in one observation are the parameters well-constrained with $\Gamma=2.1_{-2.6}^{+4.7}$ and $N_{\mathrm{H}}=$ $25_{-21}^{+57} \times 10^{22} \mathrm{~cm}^{-2}$.

A new flare of this source was observed by INTEGRAL on Sept. 9, 2003, lasting 1.3 days (Sguera et al. 2006). The 20-60 keV peak flux was $40 \mathrm{mCrab}$, and they fitted the spectrum with a combination of a black body and a power-law $\left(K T=0.7_{-0.1}^{+0.3} \mathrm{keV}\right.$ and $\left.\Gamma=2.5 \pm 0.2\right)$. Due to the shortness of the flare, they classified this source as a candidate SFXT. This tentative classification has already been pointed out by in' $\mathrm{Z}$ Zand (2005) and references therein. Grebenev \& Sunyaev (2007) discussed one possible mechanism that could trigger such bright and fast flare in this system. If the source is a supergiant windfed accreting pulsar, the propeller mechanism would inhibit the accretion when the neutron star's spin period is less than the critical value $P_{\mathrm{s}}^{*} \sim 3 \mathrm{~s}$. However, an increase in the local stellar wind density of the order of $\left(P_{\mathrm{s}}^{*} / P_{\mathrm{s}}\right)^{7 / 3}$ during a change in the stellar outflow would result in the observed flare. Kuulkers et al. (2007) also report INTEGRAL detections in the periods Feb.-Apr. in 2005 and 2006 and non detection in 2005 Aug.Oct. with an average $20-60 \mathrm{keV}$ flux of $<2 \mathrm{mCrab}$ in both detections ${ }^{1}$. Analysing all INTEGRAL public data and using the data available on the public page of the INTEGRAL Galactic Bulge program $^{1}$, Zurita Heras et al. (2007) discovered a likely period of $\sim 185$ days as the source was detected by INTEGRAL at a level of $\gtrsim 5 \sigma$ for a few days during March and September each year between 2003-2007.

Following this announcement, Swift performed a target of opportunity (ToO) observation of $5 \mathrm{ks}$ on March 30, 2007 $\left(\right.$ Romano et al. 2007) ${ }^{2}$. They detected a bright source at the position RA $(2000)=17^{\mathrm{h}} 49^{\mathrm{m}} 06.8^{\mathrm{s}}$ and $\mathrm{Dec}=-27^{\circ} 32^{\prime} 30.6^{\prime \prime}$ (6.3" at $90 \%$ confidence), 51" away from the ASCA position ${ }^{3}$. Its spectrum could be fitted with an absorbed power-law $\left(N_{\mathrm{H}}=\right.$ $\left.23_{-10}^{+14} \times 10^{22} \mathrm{~cm}^{-2}, \Gamma=2.5_{-1.7}^{+2.0}\right)$ and the observed $2-10 \mathrm{keV}$ flux is $\sim 10^{-11} \mathrm{erg} \mathrm{cm}^{-2} \mathrm{~s}^{-1}$. The Swift/UVOT telescope did not detect any optical counterpart with a $3 \sigma$ upper limit of $V=$ 20.67 mag. Within the error circle, Romano et al. (2007) report three 2MASS candidate counterparts whose infrared (IR) magnitudes in the $J H K$ bands suggest a strong optical extinction of

\footnotetext{
${ }^{1}$ See the public page of the INTEGRAL Galactic Bulge program at http://isdc. unige.ch/Science/BULGE for further information.

${ }^{2}$ From the same observation, Kong (2007) also report a slightly more accurate position with RA $(2000)=17^{\mathrm{h}} 49^{\mathrm{m}} 06.8^{\mathrm{s}}$ and Dec $=$ $-27^{\circ} 32^{\prime} 31.5^{\prime \prime}$ ( $3.8^{\prime \prime}$ at $90 \%$ confidence). He also fitted the spectrum with an absorbed power-law with $N_{\mathrm{H}}=(19 \pm 9) \times 10^{22} \mathrm{~cm}^{-2}, \Gamma=$ $2.1 \pm 1.2$, and an absorbed 2-10 keV flux of $\sim 6 \times 10^{-12} \mathrm{erg} \mathrm{cm}^{-2} \mathrm{~s}^{-1}$.

3 The distance was wrongly given in the ATel as their ASCA position does not correspond to the one published in Sakano et al. (2002).
}

20 mag. Only one candidate is compatible with a supergiant. Thus, the nature of AX J1749.1-2733 remains a mystery.

$X M M-N e w t o n$ also performed a ToO observation of the source during the expected outburst of 2007 March. From this observation, Karasev et al. $(2007,2008)$ reported the discovery of a pulsation of $\sim 132 \mathrm{~s}$. The pulse profile displays a doublepeak structure with a pulse fraction of $\sim 30 \%$ in the $2-10 \mathrm{keV}$ energy range. They also detected a pulsation during the outburst detected by INTEGRAL on Sep. 8-10, 2003 in the 20-60 keV energy range with a higher pulse fraction of $50 \%$.

Here we report multiwavelength observations performed on AX J1749.1-2733 with INTEGRAL, XMM-Newton, and the ESO/NTT telescope. In Sect. 2, we first describe the observations and the data analysis of each instrument. Then, we present the results in Sect. 3. Finally, we finish with a discussion and the conclusion on the nature of the source in Sect. 4.

\section{Observations and data analysis}

The present work is based on data of two high-energy space missions, INTEGRAL (Winkler et al. 2003) and XMM-Newton (Jansen et al. 2001), of the European Space Agency (ESA). Multi-wavelength follow-up observations were also performed with the $\mathrm{ESO}^{4} 3.5 \mathrm{~m}$ New Technology Telescope (NTT) at La Silla Observatory, Chile.

\subsection{INTEGRAL}

The INTErnational Gamma-Ray Astrophysics Laboratory (INTEGRAL) is a hard X-ray and $\gamma$-ray spacecraft $(\mathrm{S} / \mathrm{C})$ laboratory operating since Oct. 2002. The scientific payload is composed of four instruments. However, only data from the hard X-ray and soft $\gamma$-ray coded-mask imager IBIS/ISGRI (15 keV-1 MeV) (Ubertini et al. 2003; Lebrun et al. 2003) are going to be considered in this work. The imager possesses a wide field of view (FOV) of $29^{\circ}$ square with a spatial resolution of $12^{\prime}$.

The source is located near the galactic centre at a distance of $1.6^{\circ}$. As the galactic centre is one of the major scientific goals of INTEGRAL, the source's field has been extensively observed. All public data available in March 2007 for AX J1749.1-2733 have been considered in this work. Only pointings where the source is located less than $14^{\circ}$ from the FOV centre and whose time exposure is longer than $600 \mathrm{~s}$ are kept. In total, we collected 4759 pointings distributed in 129 revolutions of the $\mathrm{S} / \mathrm{C}$ that goes from Feb. 2003 (revolution 46, MJD 52 698.0) to Oct. 2005 (revolution 370, MJD 53670.1). The total exposure time on the source is $10.8 \mathrm{Ms}$ (i.e. 125.4 days) spanning 2.5 years of observations. They are not equally distributed along this period for scheduling reasons.

The ISGRI data were reduced using the Offline Scientific Analysis $\left(\mathrm{OSA}^{5}\right)$ version 6.0 software that is publicly released by the INTEGRAL Science Data Centre (ISDC) (Courvoisier et al. 2003). Individual sky images for each pointing were produced in the energy band 22-50 keV. Sky mosaics with longer exposures were built combining pointings in which the source is not detected at a $5.1 \sigma$ level or higher in individual pointings. The light curves were built using the imaging products. The source count rate was extracted with help of the tool mosaic_spec (version 1.4) that is part of the OSA package. Detections of the source in mosaics are considered at a $6 \sigma$ level or higher. We extracted a spectrum of the source during the first bright flare

\footnotetext{
${ }^{4}$ European Southern Observatory.

5 OSA is available at http://isdc. unige.ch/?Soft+download
} 
detected with ISGRI. The spectral extraction was performed using the recently released OSA version 7.0. The source spectrum was extracted with $\mathrm{i}$ i_spectra_extract within OSA for each pointing. Then, each individual spectra was summed to build one single spectrum of the source using the OSA tool spe_pick. The redistribution matrix file (RMF) isgr_rmf_grp_O025.fits was rebinned into 5 channels spread between 15 and $80 \mathrm{keV}$. Light curves with a binning of $10 \mathrm{~s}$ of the first bright flare observed with ISGRI were also extracted with ii_light version 8.2. The light curves are given in counts $\mathrm{s}^{-1}$. The $22-50 \mathrm{keV}$ count rates can be converted into other units with the relation $1 \mathrm{Crab}=117$ counts $\mathrm{s}^{-1}=9 \times 10^{-9} \mathrm{erg} \mathrm{cm}^{-2} \mathrm{~s}^{-1}$. The time unit IJD corresponds to MJD = IJD + 51544 .

\subsection{XMM-Newton}

The main scientific instrument onboard the X-ray Multi-Mirror Mission (XMM-Newton) satellite is the EPIC camera composed of two MOS (Turner et al. 2001) and one pn (Strüder et al. 2001) CCD cameras. It has imaging, timing, and spectral capabilities in the $0.2-12 \mathrm{keV}$ energy range with a $30^{\prime}$ FOV. The EPIC cameras were operating in imaging science mode with full window for MOS1, large window for MOS2 and pn, and with medium filters for each camera.

AX J1749.1-2733 was observed with XMM-Newton on March 31, 2007, from 08:03:37 to 11:27:40 UTC (MJD 54 190.337-54190.478) for a total exposure of $12.2 \mathrm{ks}$. There was no simultaneous observation with INTEGRAL.

Event lists for each MOS and pn camera were generated with the Science Analysis Software $\left(\mathrm{SAS}^{6}\right)$ version 7.0.0 using the emproc and epproc tools, respectively. The event lists were corrected for enhanced background features observed at energies higher than $10 \mathrm{keV}$, disregarding time lapses when count rates exceeded 0.52 counts $\mathrm{s}^{-1}$ for $\mathrm{pn}$ and 0.2 counts $\mathrm{s}^{-1}$ for both MOS. Therefore, the net exposure is 5.6 out of $10 \mathrm{ks}$ exposure for pn, 10.8 out of $12 \mathrm{ks}$ for MOS1, and 11.1 out of $12 \mathrm{ks}$ for MOS2. Images for MOS[12] and pn were generated with $2^{\prime \prime}$ and 4 " resolution, respectively, using good events until the quadruple level in the $0.8-10 \mathrm{keV}$ energy range and disregarding bad pixels. An accurate X-ray position determined with EPIC was calculated with the SAS task edetect_chain. Four images with energy ranges $0.5-2,2-4.5,4.5-7.5$, and 7.5-12 keV for each MOS and pn camera were extracted. Then, the best position for each individual EPIC camera was determined. Finally, the best source position was calculated as the mean of the positions given by the three cameras. The source location precision is limited by the astrometry of the $\mathrm{S} / \mathrm{C}$, that is of $2^{\prime \prime 7}$, the statistical error of $0.1^{\prime \prime}$ being insignificant in comparison.

In the EPIC/pn image, one bright source was detected in the CCD\#1 near the read-out node. The event list of the source+background was extracted from a region of $35^{\prime \prime}$ radius centred on the source. A background event list was extracted in the same CCD at same distance of the read-out node from a region of similar size not affected by the bright source. Spectra were extracted by selecting single+double events but disregarding bad pixels. Specific RMF and ancillary response files (ARF) were generated with the SAS tasks rmfgen and arfgen, respectively. The Xspec version 11.3.2t package (Arnaud 1996) was used to plot and fit the resulting spectra corrected from

\footnotetext{
6 SAS is available at http://xmm.vilspa.esa.es/external/ xmm_sw_cal/sas.shtml

7 The XMM-Newton astrometric accuracy is described in the calibration document XMM-CAL-TN-QQ18.
}

background. For light curves, single and double events were also selected within the same regions defined in the spectral step. The source light curves were corrected from the background using the SAS task lccorr, and we applied the barycentric correction with the SAS task barycen.

\subsection{ESO/NTT}

Multiwavelength observations were undertaken soon after the accurate X-ray position was given by Romano et al. (2007) and Kong (2007). The observations were achieved with the $3.5 \mathrm{~m}$ ESO/NTT telescope in two domains: in the near IR (NIR) domain $(1-2.4 \mu \mathrm{m})$ with the spectro-imager SOFI and in the optical domain with the imager SUSI-2 (350-900 nm). The observations were carried out as part of the ToO programme 079.D-0432(A) (PI: S. Chaty), through service mode. Astrometry, photometry, and spectroscopy were achieved during those observations. The spectroscopy was performed on the bright counterpart proposed by Romano et al. (2007) (2MASS source \#1).

\subsubsection{NIR observations}

The NIR photometry in the bands $J, H$, and $K_{\mathrm{S}}$ was performed on April 3, 2007, with the spectro-imager SOFI installed on a Nasmyth focus of the NTT. The observations were centred on the known X-ray position of AX J1749.1-2733. The large imaging mode was used during those observations, and it gives an image pixel scale of $0.288^{\prime \prime}$ and an FOV of $4.92^{\prime} \times 4.92^{\prime}$. Nine images were taken for each band with integration times of $60 \mathrm{~s}$ for $J$ and $47.3 \mathrm{~s}$ for $H$ and $K_{\mathrm{s}}$. For each band, four of the nine observations were taken with a slight offset of $\sim 30^{\prime \prime}$ that allowed us to build the NIR sky to subtract it from the images. Six standard stars chosen from the faint NIR standard star catalogue (Persson et al. 1998) were also observed: S262-E, S495E, S708-D, S264-D, P565-C, and S875-C. Five observations per band were performed on each standard star. The first observation was centred on the target and then, the next four images were taken with an offset of $\sim 45^{\prime \prime}$ compared to the first one.

\subsubsection{Optical observations}

The optical observations were carried out on April 6, 2007, between UTC 08h40 and 08h40 with the imager SUSI-2 also installed on the same Nasmyth focus of the NTT as SOFI. Optical photometry in $U, B, V, R, I$, and $Z$ bands was obtained. The FOV was $5.5^{\prime} \times 5.5^{\prime}$ with a binning of factor 2 that gives a pixel scale of $0.161^{\prime \prime}$ per pixel. The exposure time was $60 \mathrm{~s}$ for each filter. Nine photometric standards (selected in the optical standard star catalogue of Landolt 1992) were also observed in the fields PG 1657+078 and PG 1633+099. The integration times in each filter varied between $10-100 \mathrm{~s}$.

\subsubsection{Data reduction}

The reduction of both optical and NIR data was performed with the Image Reduction and Analysis Facility (IRAF ${ }^{8}$ ) version 2.13 beta2. Data reduction was performed using standard procedures on the optical and NIR images, including the correction of the dark current, the flat-fielding, and NIR sky subtraction.

We performed accurate astrometry on each image ( $U, B, V, R, I, Z, J, H, K_{\mathrm{S}}$ ) using the gaia tool from the Starlink

${ }^{8}$ IRAF is available at http $: / /$ iraf.net/ 


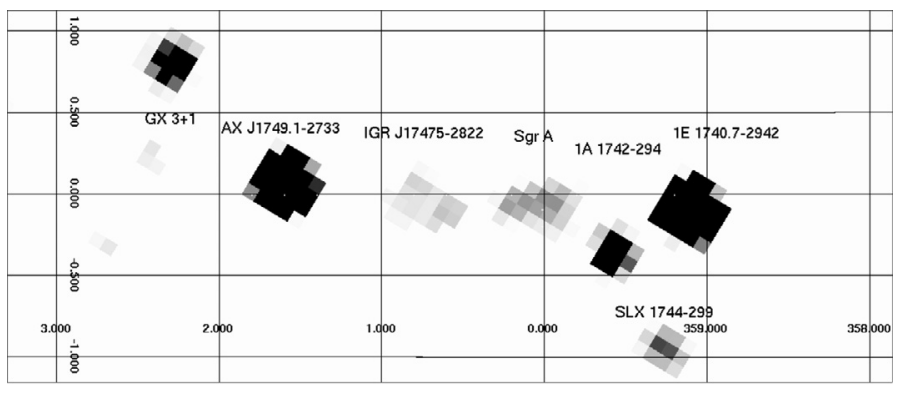

Fig. 1. ISGRI $22-50 \mathrm{keV}$ significance map built with the pointings of rev. 110 listed in Table 1 corresponding to the first outburst of AX J1749.1-2733 observed with INTEGRAL. AX J1749.1-2733 is located near the galactic centre, and it is detected with a significance of $44 \sigma$ for an exposure of $51.3 \mathrm{ks}$. The coordinate grid corresponds to the galactic longitude and latitude $l, b$ in degrees.

suite $^{9}$ and using the 2MASS catalogue for the NIR images and the USNO B1.0 catalogue for the optical images. The NIR rms of astrometry fit was always lower than $0.4^{\prime \prime}$ with the expected pixel scale in $x, y$ axis of $-0.288 \times 0.288^{\prime \prime} /$ pixel. The optical rms of astrometry fit was also lower than $0.4^{\prime \prime}$ with the expected pixel scale in $x, y$ axis of $0.161 \times 0.161^{\prime \prime} /$ pixel.

Then, we carried out aperture photometry in a crowded field using the IRAF digiphot. daophot package. The instrumental magnitudes $m_{\text {instr }}$ were transformed into apparent magnitudes $m_{\text {app }}$ using the standard photometric relation: $m_{\text {app }}=m_{\text {instr }}-Z_{\mathrm{p}}-$ ext $\times A M$, where $Z_{\mathrm{p}}$ is the zero-point, ext the extinction, and $A M$ the airmass. The colour term was not used because 1) there were not enough standard stars in NIR and 2) in optical bands, we did not detect the sources of interest in several bands (particularly in $V$ ) in order to perform colour correction. The $Z_{\mathrm{p}}$ and ext parameters were fitted using the photometric relation in order to match the instrumental and apparent magnitudes of the standard stars. For the optical photometry, the extinction parameters were fixed to the values given during the SUSI- 2 calibration ${ }^{10}$. The airmass, zero-point, and extinction parameters are reported at the end of Table 4.

\section{Results}

\subsection{Refined X-ray position}

A $22-50 \mathrm{keV}$ image was generated from the first outburst whose pointings are listed in Table 1 . The source is detected at a level of $44 \sigma$ for a total exposure of $51.3 \mathrm{ks}$ (see Fig. 1).

The best hard X-ray position is RA $(2000)=17^{\mathrm{h}} 49^{\mathrm{m}} 05.5^{\mathrm{s}}$ and Dec $=-27^{\circ} 32^{\prime} 38.4^{\prime \prime}$ with an error of $1.8^{\prime}$ related to its significance (Gros et al. 2003). This position is consistent with the position previously reported in the 3rd ISGRI catalogue (Bird et al. 2007) and in Sguera et al. (2006).

One bright source lies within the AX J1749.1-2733 ISGRI error circle in EPIC images (see Fig. 2). The best position determined with EPIC is RA $(2000)=17^{\mathrm{h}} 49^{\mathrm{m}} 06.8^{\mathrm{s}}$ and Dec $=$ $-27^{\circ} 32^{\prime} 32.5^{\prime \prime}$ with a systematic uncertainty of $2^{\prime \prime}$. This X-ray refined position is consistent with the respective error circles of ASCA, INTEGRAL, and Swift. There is only a difference of $1^{\prime \prime}$ between the Swift and the EPIC positions.

\footnotetext{
9 Thanks to the Joint Astronomy Centre, the Starlink software collection is still available at http://starlink. jach.hawaii . edu/ 10 retrieved at http://www.ls.eso.org/lasilla/sciops/ntt/ susi/archive/susi2zp.dat
}

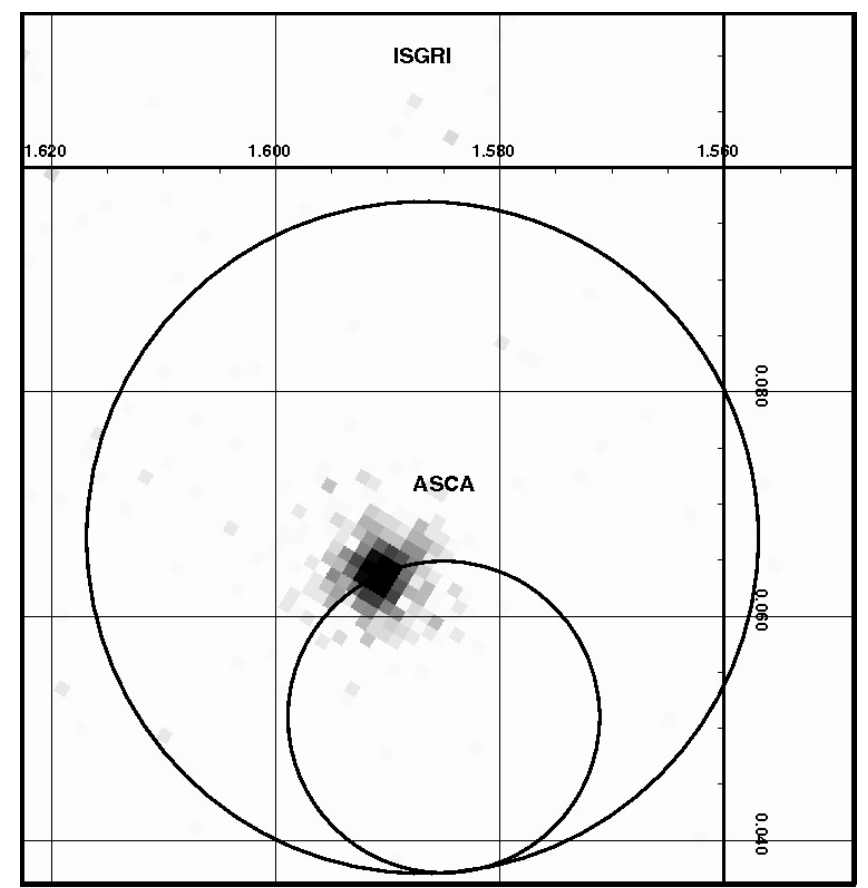

Fig. 2. EPIC/MOS1 image in the $0.8-10 \mathrm{keV}$ energy range. The ISGRI (derived from the first outburst) and ASCA error circles are reported, with the Swift position embedded in the bright source. The best EPIC position is RA $(2000)=17^{\mathrm{h}} 49^{\mathrm{m}} 06.8^{\mathrm{s}}$ and Dec $=-27^{\circ} 32^{\prime} 32.5^{\prime \prime}$ with a systematic uncertainty of $2^{\prime \prime}$ (e.g. galactic $l, b=1.5908^{\circ}, 0.0637^{\circ}$ ). The coordinate grid corresponds to the galactic longitude and latitude $l, b$ in degrees.

\subsection{Timing analysis}

While the long-term variability of AX J1749.1-2733 can be studied with the huge amount of data collected with INTEGRAL, $X M M-N e w t o n$ allows study of the short-term variability of the source.

\subsubsection{Long-term variability}

The long-term variability of AX J1749.1-2733 was studied using the ISGRI data in the energy range $22-50 \mathrm{keV}$. All detections within one pointing of the $\mathrm{S} / \mathrm{C}$ indicating an increase in the hard X-ray emission on a short time-scale $(\lesssim 1 \mathrm{~h})$ were searched. In total, the source was detected at a level higher than $5.1 \sigma$ in only 16 pointings out of the 4759 available (see Table 1). Two bright flares were observed during the $2.5 \mathrm{yr}$ observations, one lasting $\lesssim 1$ day in revolution $110^{11}$ (see Fig. 3) and another short one in revolution 173 lasting $1 \mathrm{~h}$. In revolution 110, the source was detected in two single pointings at a level slightly higher than the detection threshold before displaying a bright flare that started at IJD 1347.3636 and lasts $\sim 0.5$ days. The count rate's increase/decrease was rather smooth. The peak intensity was reached in the middle of the outburst with two local maxima at $7.5-7.8 \pm 0.5$ counts $\mathrm{s}^{-1}$ and a small decay in between. The two flares are also reported in the long-term light curve (see Fig. 4).

Then, detections on a larger timescale are searched. The long-term monitoring of AX J1749.1-2733 is summarised in Table 2. We built mosaics for each revolution with the source within the FOV. Concerning revolutions 110 and 173, the

\footnotetext{
11 This one is reported in Sguera et al. (2006).
} 
Table 1. List of detections of AX J1749.1-2733 in single pointings with IBIS/ISGRI.

\begin{tabular}{cccccc}
\hline \hline $\begin{array}{c}\text { Time } \\
\text { IJD }\end{array}$ & $\begin{array}{c}22-50 \mathrm{keV} F l u x^{-} \\
\text {counts s }^{-1}\end{array}$ & $\begin{array}{c}\text { Error flux } \\
\text { counts s }^{-1}\end{array}$ & Sigma & $\begin{array}{c}\text { Off-axis angle } \\
\text { degrees }\end{array}$ & Pointing \\
\hline 1346.9390 & 3.6 & 0.6 & 6.4 & 3.7 & 01100038 \\
1347.2492 & 3.8 & 0.6 & 6.3 & 4.7 & 01100045 \\
1347.3421 & 3.7 & 0.5 & 7.4 & 0.8 & 01100047 \\
1347.3836 & 4.5 & 0.6 & 8.1 & 1.5 & 01100048 \\
1347.4250 & 5.2 & 0.6 & 9.2 & 3.4 & 01100049 \\
1347.4664 & 5.1 & 0.6 & 8.7 & 4.2 & 01100050 \\
1347.5078 & 5.9 & 0.6 & 10.7 & 2.9 & 01100051 \\
1347.5491 & 7.8 & 0.5 & 14.5 & 2.6 & 01100052 \\
1347.5903 & 6.3 & 0.6 & 11.4 & 3.7 & 01100053 \\
1347.6318 & 6.7 & 0.6 & 11.7 & 5.3 & 01100054 \\
1347.6732 & 7.5 & 0.6 & 13.3 & 2.7 & 01100055 \\
1347.7146 & 6.7 & 0.5 & 13.7 & 0.8 & 01100056 \\
1347.7560 & 6.4 & 0.6 & 11.5 & 1.5 & 01100057 \\
1347.7972 & 5.2 & 0.5 & 9.5 & 2.0 & 01100058 \\
1347.8385 & 4.0 & 0.5 & 7.7 & 1.6 & 01100059 \\
1534.6049 & 3.3 & 0.6 & 5.5 & 4.9 & 01730024 \\
\hline
\end{tabular}

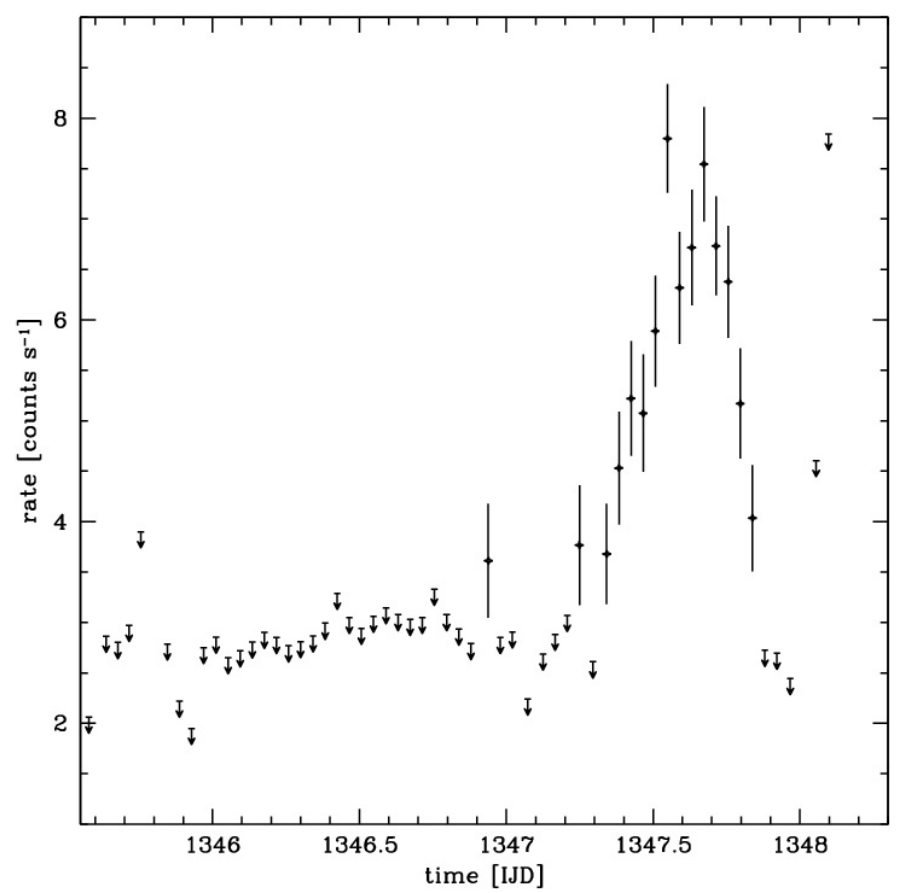

Fig. 3. ISGRI 22-50 keV light curve of AX J1749.1-2733 during rev. 110 . The $5.1 \sigma$ upper limits are reported when the source was not detected.

pointings where AX J1749.1-2733 was detected were discarded before building both mosaics. The exposures are long with 127 and $144 \mathrm{ks}$, respectively. In both cases, the source was detected significantly with $11 \sigma$ and a count rate of $1.0 \pm 0.1$ (triangle points in Fig. 4). In the other mosaics, the source was detected in 10 revolutions at a significant level of $>6 \sigma$ for exposure times between 100-200 ks (square points in Fig. 4).

All the detections are distributed in 5 outbursts that are separated by half a year each time. The two flares reported above occurred during outbursts 1 and 2 . There are 2 isolated revolutions and three periods where the source is detected in consecutive revolutions ( 3 or more) giving elapsed time of activity of $11.6,2.5,2.3,8.4$, and 8.3 days. However, the observed flux level during the outbursts is faint with rates of $(0.56-$ $1.25) \pm 0.1$ counts $\mathrm{s}^{-1}$. With such faintness, the exposure time in individual revolution needs to be higher than $\sim 100 \mathrm{ks}$ in order to detect the source. For each observed outburst except the first one, the revolutions prior to and/or after the outburst do not fill this condition. Considering the nearest revolutions with an exposure time longer than $100 \mathrm{ks}$ and surrounding the outbursts, the maximum outburst duration that we derive is $\lesssim 18 \mathrm{~d}$. Only the first outburst duration can be constrained to less than $12.4 \mathrm{~d}$, which is the difference between the ending and start of rev. 109 and 114 . Both revolution exposure times are $\sim 200 \mathrm{ks}$, so it firmly excludes any activity by AX J1749.1-2733 with the same intensity displayed in the other detections. The outbursts 1, 4, 5 all display

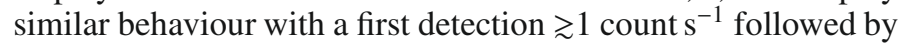
fainter detections. Considering the start of these 3 outbursts, we derive a period of $185.5 \pm 1.1 \mathrm{~d}$. The two shorter outbursts 2 and 3 are consistent with this period and an outburst's duration of $\lesssim 12$ days. The source was not detected during the first part of the observation as the expected outburst time falls in the middle of a gap of $12 \mathrm{~d}$ without observation. Moreover, the period of AX J1749.1-2733 may be shorter by a factor $185.5 / f \mathrm{~d}$ where $f=2,3$, or 4 once considered the total unequal coverage of the source field by INTEGRAL over the years. However, INTEGRAL observations discard factors $f \geq 5$.

The ASCA detections are 10 times fainter than the $X M M$-Newton ones that occurred during an outburst of the source. INTEGRAL cannot reach this faintness, as detected by $A S C A$, even for exposure of several Ms. They fall at phases $0.2-$ 0.28 when the period is $185.5 \mathrm{~d}$. If the source detected by $A S C A$ is the same one detected by both INTEGRAL and XMM-Newton, either the outbursts last longer with a duration of $\sim 50 \mathrm{~d}$, with $A S C A$ observing the end of the outburst, or the period of the system is shorter. In the last case, ASCA detections would be compatible with $f=4$, making a period of $46.4 \mathrm{~d}$.

The revolutions where the source is not detected are accumulated only when they are separated by less than 30 days. This results in 11 mosaics with exposure times lying between 333$1005 \mathrm{ks}$. The $1 \sigma$ level lies between $0.04-0.07$ counts s$^{-1}(=0.05-$ $0.08 \mathrm{mCrab})$. As the field is noisier with exposure times $>300 \mathrm{ks}$, the detection limit is set to $10 \sigma$ (i.e. $\gtrsim 0.5 \mathrm{mCrab}$ ). The fluxes extracted in these mosaics are reported in the multi-revolutions part of Table 2 (upper limit arrows in Fig. 4, the observed fraction of these upper limits is reported in Table 2). The source is never detected besides the 5 outbursts discussed earlier. The detailed 22-50 keV light curve is shown in Fig. 4. 
Table 2. Long-term monitoring of AX J1749.1-2733 with INTEGRAL/IBIS/ISGRI.

\begin{tabular}{lcccccc}
\hline \hline $\begin{array}{c}\text { Time start } \\
\text { IJD }\end{array}$ & $\begin{array}{c}\text { Time stop } \\
\text { IJD }\end{array}$ & $\begin{array}{c}\text { 22-50 keV Flux } \\
\text { counts s }^{-1}\end{array}$ & $\begin{array}{c}\text { Flux error } \\
\text { counts s }^{-1}\end{array}$ & $\begin{array}{c}\text { Detection } \\
\text { sigma }\end{array}$ & $\begin{array}{c}\text { Exposure } \\
\text { s }\end{array}$ & $\begin{array}{c}\text { Revolution or } \\
\text { observed fraction }^{\dagger}\end{array}$ \\
\hline $\begin{array}{l}\text { one revolution } \\
1345.5410\end{array}$ & 1348.1174 & 1.08 & 0.10 & 11.4 & 126803 & 110 \\
1348.6103 & 1351.1086 & 0.61 & 0.08 & 7.8 & 176622 & 111 \\
1351.5239 & 1354.1001 & 0.56 & 0.07 & 7.8 & 203381 & 112 \\
1354.5150 & 1357.0919 & 0.61 & 0.07 & 8.3 & 195763 & 113 \\
1534.1522 & 1536.6650 & 0.99 & 0.09 & 10.8 & 143943 & 173 \\
1722.5068 & 1724.7996 & 0.85 & 0.10 & 8.8 & 147812 & 236 \\
1902.1512 & 1904.5188 & 1.08 & 0.12 & 9.2 & 137347 & 296 \\
1904.9797 & 1907.4973 & 0.97 & 0.08 & 11.9 & 192511 & 297 \\
1907.9482 & 1910.5226 & 0.73 & 0.09 & 8.6 & 177189 & 298 \\
2087.5924 & 2089.8853 & 1.25 & 0.15 & 8.6 & 101696 & 358 \\
2090.6097 & 2092.9742 & 0.88 & 0.11 & 8.0 & 129327 & 359 \\
2093.5752 & 2095.9587 & 0.93 & 0.14 & 6.5 & 108779 & 360 \\
multi-revolutions & & & & & & \\
1154.1922 & 1214.5034 & 0.34 & 0.04 & 7.9 & 887100 & 0.17 \\
1324.9592 & 1345.1323 & 0.21 & 0.04 & 6.0 & 1004817 & 0.17 \\
1357.5105 & 1383.3782 & 0.26 & 0.04 & 6.6 & 904838 & 0.40 \\
1507.4945 & 1530.5140 & 0.31 & 0.05 & 7.0 & 687994 & 0.35 \\
1537.7486 & 1571.5313 & 0.35 & 0.04 & 7.8 & 668850 & 0.23 \\
1690.1832 & 1722.0688 & 0.36 & 0.05 & 7.0 & 603794 & 0.22 \\
1725.8560 & 1762.0251 & 0.26 & 0.04 & 6.8 & 976316 & 0.31 \\
1873.1647 & 1900.9215 & 0.37 & 0.07 & 5.8 & 333370 & 0.14 \\
1910.9388 & 1944.1377 & 0.37 & 0.04 & 8.8 & 770675 & 0.27 \\
2055.1532 & 2086.8640 & 0.42 & 0.07 & 6.2 & 389952 & 0.14 \\
2097.9383 & 2125.8160 & 0.47 & 0.05 & 9.1 & 658682 & 0.27 \\
\hline
\end{tabular}

${ }^{\dagger}$ Either the revolution number (top) or the observed fraction derived as exposure/(time stop-time start) (bottom).

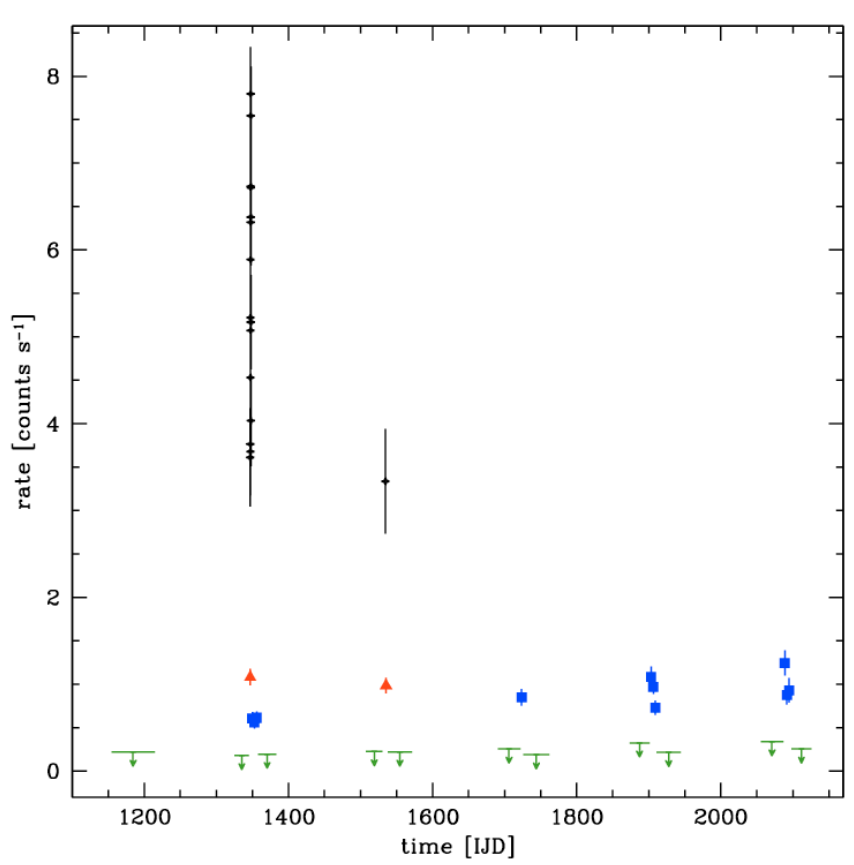

Fig. 4. ISGRI 22-50 keV long-term light curve of AX J1749.1-2733. The 2 flares reported in Table 1 are shown as vertical crosses for each single pointing. The other points correspond to average count rates extracted in large mosaic images. Details about symbols are found in the text.

\subsubsection{Short-term variability}

The short-term variability of AX J1749.1-2733 is studied using the EPIC data. The 0.4-10 keV light curve is rather constant with a low average count rate of $\sim 0.4$ counts $\mathrm{s}^{-1}$. No flare is observed. Considering the period and duration of outbursts derived from the INTEGRAL long-term light curve, the expected outburst near the XMM-Newton observation happens between MJD 54188 and 54 206. Therefore, XMM-Newton observed AX J1749.1-2733 at the beginning of one of its outburst (MJD 54 190).

We searched for the pulsation when computing the power density spectra of the EPIC light curve and of the 10 s-binned ISGRI light curve extracted during the long flare shown in Fig. 3. We used the fast computing method of the Lomb-Scargle periodogram proposed by Press \& Rybicki (1989). The uncertainty of the period is computed using Eq. (14) in Horne \& Baliunas (1986). Both periodograms are shown in Fig. 5 (left column). The maximum power is detected at $66.09 \pm 0.07 \mathrm{~s}$ and $65.789 \pm 0.009 \mathrm{~s}$ for $\mathrm{pn}$ and ISGRI, respectively. The corresponding folded light curves are also shown in Fig. 5 (right column) with zero epochs T0 of MJD 54190.391 and 52891.4. The pulse fractions, defined as $P_{\mathrm{f}}=\left(I_{\max }-I_{\min }\right) /\left(I_{\max }+I_{\min }\right)$ with $I_{\max }$ and $I_{\min }$ being the maximum and minimum of the intensities of the folded light curve, reach $22 \pm 6$ and $29 \pm 11 \%$. The pulse profiles show one broad peak for pn and a slightly narrower one for ISGRI. As both pulsations are similar over a period of $3.6 \mathrm{yr}$, the timing analysis confirms that INTEGRAL and XMM-Newton detected the same source, as it is unlikely that $2 \mathrm{X}$-ray sources with the same pulsation could be located in such a small box of a few arcseconds.

A pulsation of $132 \mathrm{~s}$ was also reported by Karasev et al. (2007, 2008) using the same XMM-Newton data presented here, along with the ISGRI data corresponding to the bright flare detected by INTEGRAL. No significant power appears at $\sim 132 \mathrm{~s}$ in both periodograms in Fig. 5. In the pn periodogram, the closest stronger signals appear at 123 and $139 \mathrm{~s}$; yet their power are twice lower than the one at $66 \mathrm{~s}$, and they do not appear in 


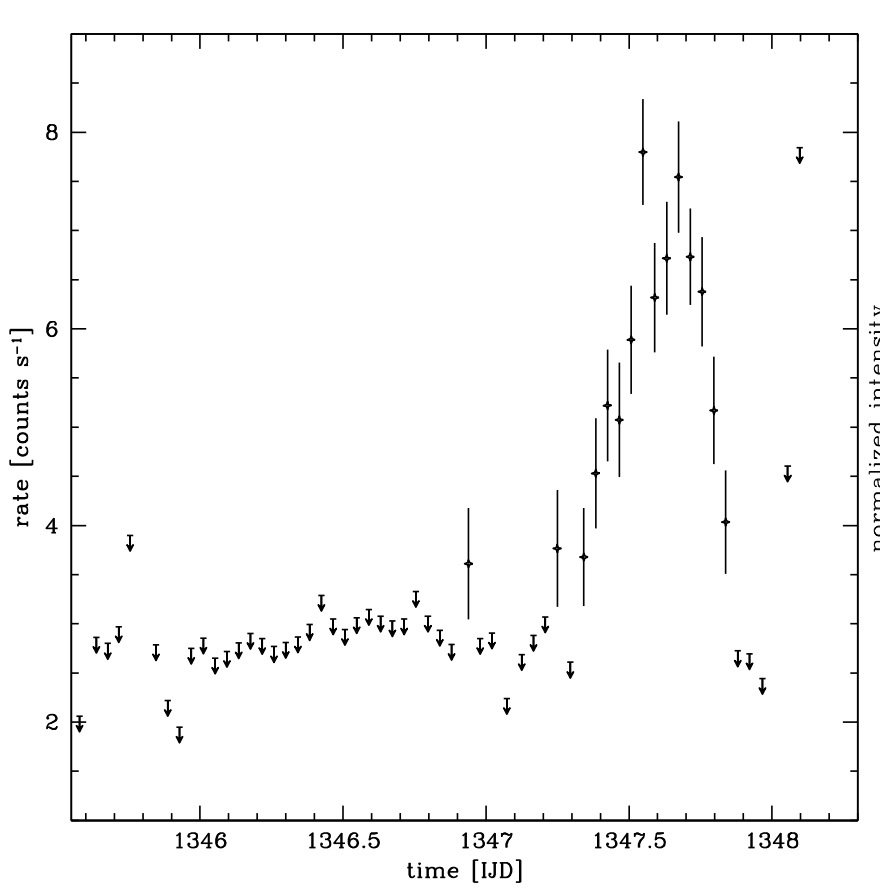

(a) EPIC/pn periodogram

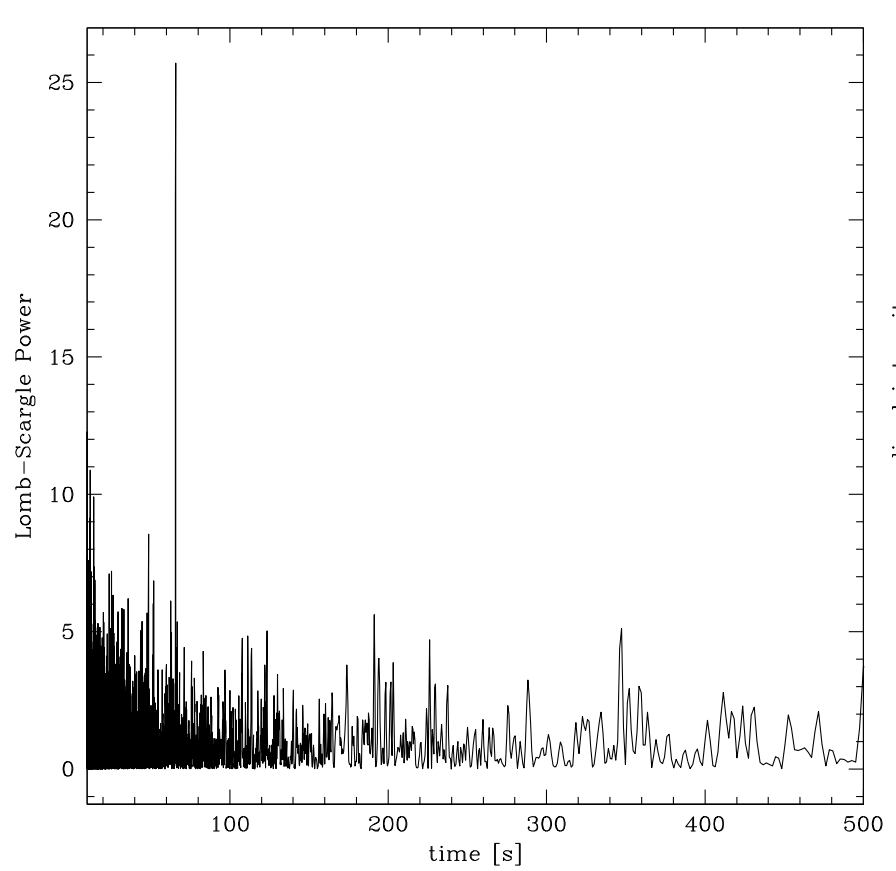

(c) ISGRI periodogram

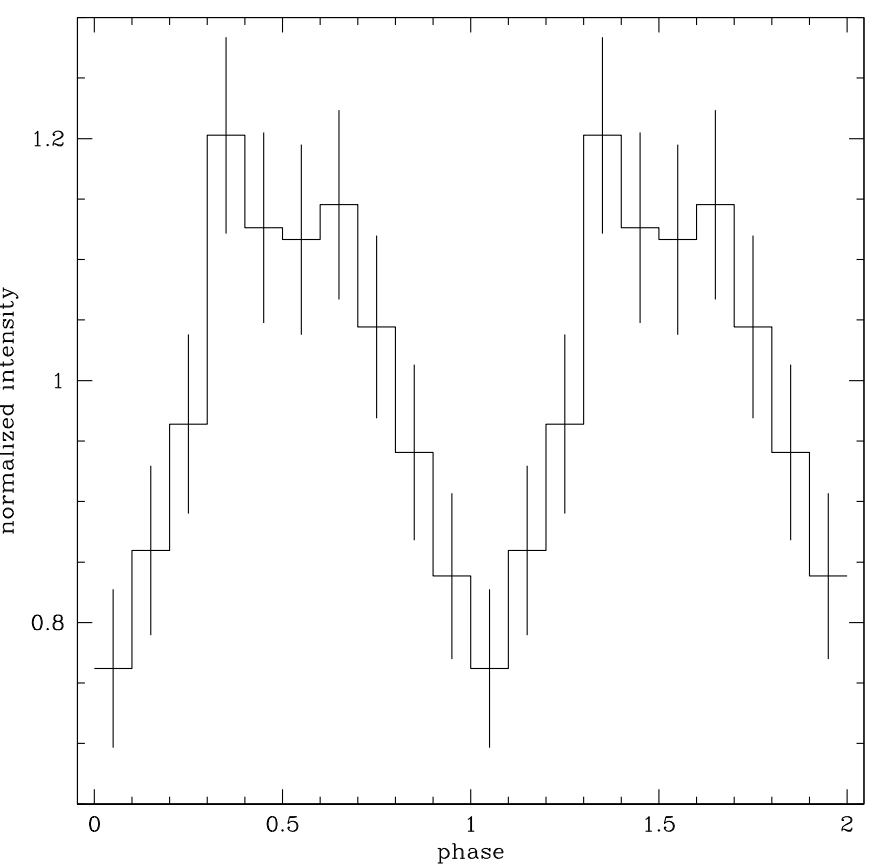

(b) pn folded light curve, $P=66.09 \pm 0.07 \mathrm{~s}$ and T0=MJD 54190.391

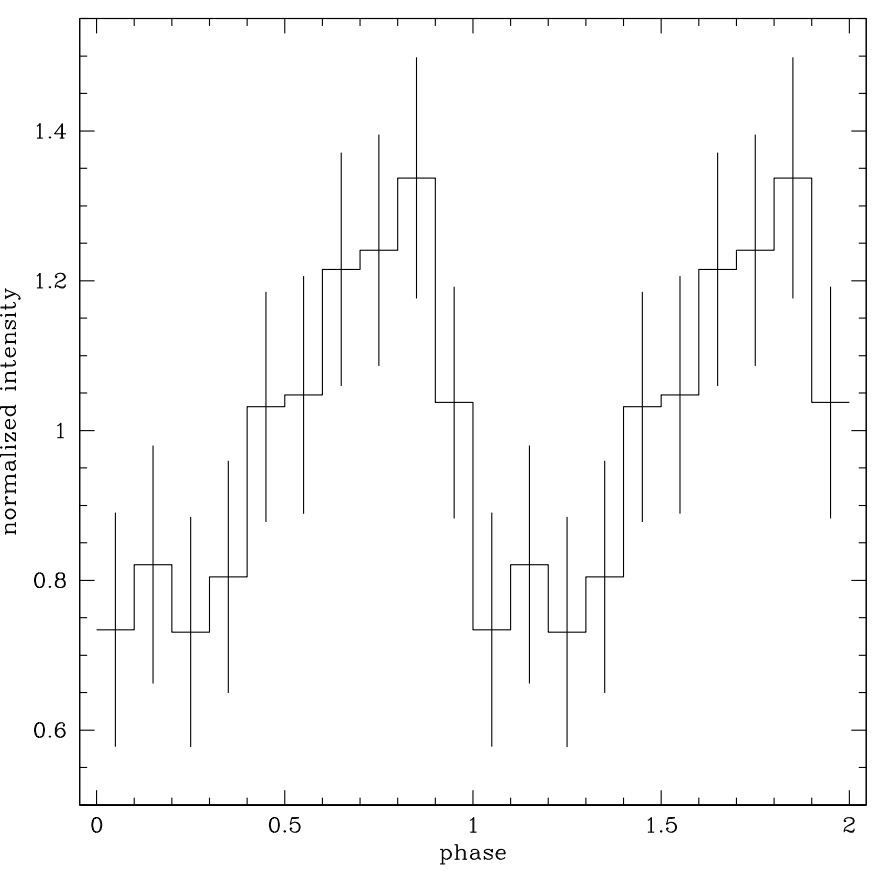

(d) ISGRI folded light curve, $P=65.789 \pm 0.009 \mathrm{~s}$ and T0=MJD 52891.4

Fig. 5. Periodograms and folded light curve for EPIC/pn (top, $0.4-10 \mathrm{keV}$ ) and ISGRI (bottom, 22-50 keV). The maximum power corresponds to $66.09 \pm 0.07 \mathrm{~s}$ and $65.789 \pm 0.009 \mathrm{~s}$, respectively. The folded light curves use the derived periods and zero epochs of MJD 54190.391 and 52891.4.

the ISGRI periodogram. Folding the light curve at twice the period we derived show a double-peak profile in both folded light curves. Still, both local peaks show similar shape and intensity. Therefore, the pulsation around $66 \mathrm{~s}$ is likely to be the real pulsation, while the $132 \mathrm{~s}$ one is its first harmonic. Karasev et al. (2008) shows that no difference is observed in the pulse profile derived with ISGRI when folding the light curve with either 66 or $132 \mathrm{~s}$. On the other hand, they show a slight difference between both pulse profiles derived with EPIC/pn, but this difference is not significant within the error bars.

\subsection{Spectral analysis}

The broad-band XMM-Newton-INTEGRAL X-ray spectrum is shown in Fig. 6. The X-ray spectrum extracted with pn is combined with the hard X-ray one extracted during the bright flare seen in ISGRI. Therefore, an important constant factor must be applied, because the intensity difference of AX J1749.1-2733 between the 2 observations is large, and it may correspond to two different states of the source. Still, the combined spectrum will constrain the spectral model that will fit the continuum shape 


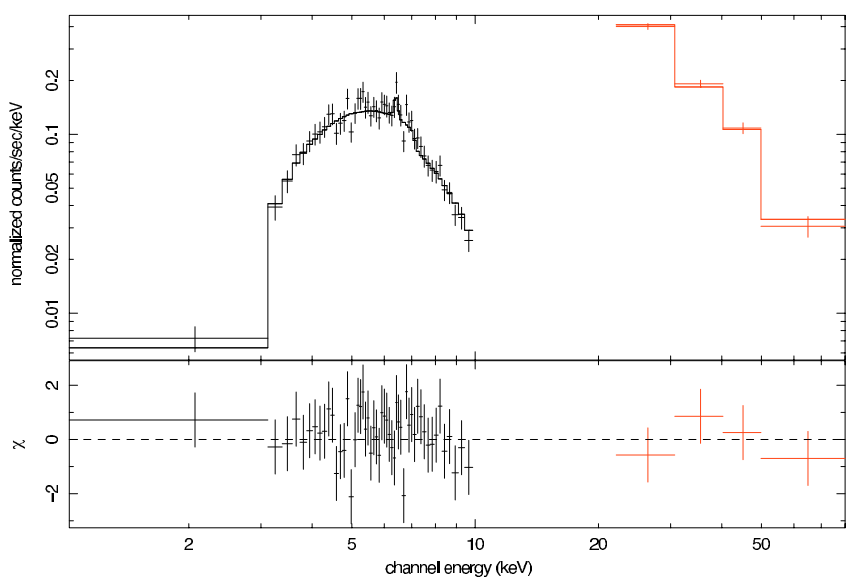

Fig. 6. High-energy broad-band spectrum of AX J1749.1-2733, combining the EPIC/pn and IBIS/ISGRI spectra. The best-fit model (absorbed PL with high-energy cutoff and an additional emission line) and residuals are also shown.

Table 3. Best-fitting results for the broad-band spectrum.

\begin{tabular}{lcl}
\hline \hline Parameters & Values & Unit \\
\hline$N_{\mathrm{H}}$ & $20.1_{-1.3}^{+1.5}$ & $10^{22} \mathrm{H} \mathrm{cm}^{-2}$ \\
$\Gamma$ & $1.0_{-0.3}^{+0.1}$ & \\
$E_{\text {cut }}$ & $21_{-3}^{+5}$ & $\mathrm{keV}$ \\
$C_{\text {ISGRI }}$ & 18 & \\
$\chi_{v}^{2}$ (d.o.f.) & 1.03 & $(105 \mathrm{~d} . \mathrm{o} . \mathrm{f})$. \\
unabs. 2-10 keV Flux & 0.2 & $10^{-10} \mathrm{erg} \mathrm{cm}^{-2} \mathrm{~s}^{-1}$ \\
unabs. 22-50 keV Flux & 3.2 & $10^{-10} \mathrm{erg} \mathrm{cm}^{-2} \mathrm{~s}^{-1}$ \\
unabs. 0.2-100 keV Flux & 6.9 & $10^{-10} \mathrm{erg} \mathrm{cm}^{-2} \mathrm{~s}^{-1}$ \\
\hline
\end{tabular}

Note: The model is cons"wabs*cutoffpl. The unabsorbed flux is computed setting $N_{\mathrm{H}}=0$.

of the source. The X-ray spectral bins are grouped to possess at least 30 counts per channel, allowing use of the $\chi^{2}$ statistic.

First, the spectrum shows a strong absorption at low energies. Simple phenomenological models, such as absorbed black body (BB) or power-law (PL), fail to fit the data with $\chi_{v}^{2}=3.4$ or 1.7 (each 106 d.o.f.), respectively. Disregarding the BB model, the absorbed PL clearly needs a high-energy cutoff to fit the data. The fit strongly improves with $\chi_{v}^{2}=1.03$ (105 d.o.f.). We also explore the possibility of a line at $6.4 \mathrm{keV}$ where some excess is visible. The tentative detection provides a good fit $\left(E_{\text {line }}=6.41_{-0.07}^{+0.08} \mathrm{keV}, F_{\text {line }}=(1.3 \pm 0.9) \times 10^{-5} \mathrm{ph} \mathrm{cm}^{-2} \mathrm{~s}^{-1}\right.$, $\left.E W_{\text {line }}=52.5 \mathrm{eV}\right)$ of $\chi_{v}^{2}=0.99(103$ d.o.f. $)$, but this is not significant because the line detection is $<3 \sigma$. The possibility of an excess at low energies in addition to the absorption was also studied. However, the addition of a component at low energies is not significant as shown by the high value of the F-test probability of 0.1 . The best-fit parameters are listed in Table 3 .

\subsection{Identifying the optical/NIR counterpart}

The field around AX J1749.1-2733 is very crowded, so it possesses several counterpart candidates within the error circles of the previously reported high-energy missions. In Fig. 7, we show the field in the $J$ band. With the higher accuracy of Swift, 3 2MASS candidates were reported (Romano et al. 2007). Still, with SOFI, we note that there are 11 candidates in the $J$ band

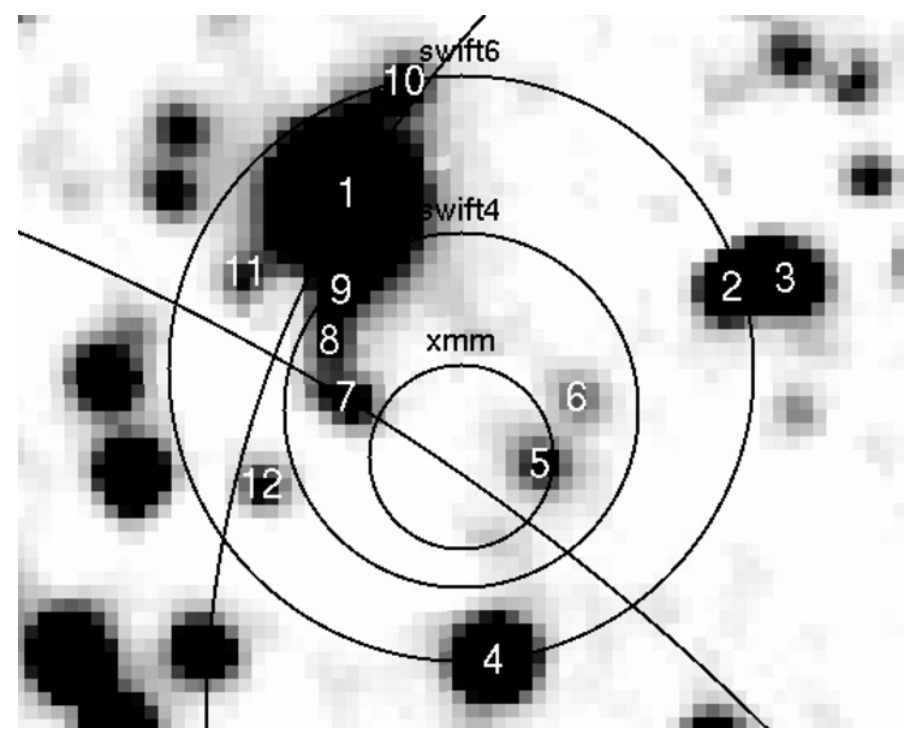

Fig. 7. SOFI image in filter $J$. The 12 candidates within the large Swift error circle (6") are reported. Only candidate \#5 lies within the accurate $\mathrm{X}$-ray position of XMM-Newton $\left(2^{\prime \prime}\right)$.

within this 6" Swift box ${ }^{12}$. With the improved Swift position given by Kong (2007), the 3 2MASS counterparts are ruled out, but 5 faint candidates remain. Taking the best X-ray position given by XMM-Newton into account (see Sect. 3.1), only candidate \#5 remains as a possible optical/NIR counterpart to AX J1749.1-2733. The source was not detected in filters $U, B$, and $V$, but a faint source was detected in the other filters as highlighted in Fig. 8. The photometry of all sources is reported in Table 4.

\section{Discussion and conclusion}

The source possesses a long orbital period of $(185.5 \pm 1.1) / f \mathrm{~d}$ where $f=1,2,3$, or 4 and a spin period of $\sim 66 \mathrm{~s}$. At the likely periastron passage, the source goes through an outburst whose duration is estimated to $\sim 12 \mathrm{~d}$ (with an upper-limit of $\lesssim 18 \mathrm{~d}$ ). With such orbital period $(f=1)$ and outburst duration, the source is bright $<10 \%$ of its time. The recurrence of such long outbursts related to the binary motion corresponds to type I outbursts observed in other BeXB (Coe 2000; Negueruela 2007). Furthermore, combining the observed pulsation and orbital period, AX J1749.1-2733 falls well in the BeXB arm of the Corbet diagram (i.e. $P_{\text {spin }}$ vs. $P_{\text {orb }}$; Corbet 1984,1986$)$ strengthening its identification as a BeXB. The large orbital period rules out the possibility to associate this source with a low-mass X-ray binary whose companion is a star of the main sequence. Flaring activity in BeXB has never been observed except in EXO 2030+375 and recently in SWIFT J1626.6-5156 (Reig et al. 2008, and references therein). For SWIFT J1626.6-5156, the intensity varied by a factor 4 on a timescale of $450 \mathrm{~s}$ with sharp rise/decay of the count rate. This behaviour differs from the bright flare of AX J1749.1-2733 detected with ISGRI in revolution 110, which shows a smooth rate's rise/decay, a variability factor of 8 , and a duration of $0.5 \mathrm{~d}$. This bright flare in revolution 110 is likely to be due to a sensitivity bias where only the peak flux of the outburst is significantly detected in individual pointings. However, flaring activity cannot be discarded for AX J1749.1-2733

\footnotetext{
12 The 3 2MASS candidates correspond to sources \#1, \#4 and a blended source constituted of \#2 and \#3.
} 
Table 4. Optical and NIR photometry of the candidate counterparts located within the large Swift error circle.

\begin{tabular}{cccccc}
\hline \hline Candidate $^{\dagger}$ & $R$ & $I$ & $J$ & $H$ & $K_{\mathrm{s}}$ \\
\hline$\# 1$ & $22.6 \pm 0.5$ & $19.68 \pm 0.04$ & $12.49 \pm 0.01$ & $10.54 \pm 0.01$ & $9.18 \pm 0.01$ \\
$\# 2$ & - & - & $16.32 \pm 0.06$ & $14.62 \pm 0.04$ & $13.51 \pm 0.04$ \\
$\# 3$ & - & - & $15.84 \pm 0.04$ & $14.23 \pm 0.03$ & $13.27 \pm 0.05$ \\
$\# 4$ & - & - & $15.27 \pm 0.01$ & $13.54 \pm 0.01$ & $12.45 \pm 0.02$ \\
$\# 5^{\ddagger}$ & $21.3 \pm 0.1$ & $20.30 \pm 0.09$ & $17.42 \pm 0.03$ & $16.71 \pm 0.02$ & $15.75 \pm 0.07$ \\
$\# 6$ & - & - & $18.08 \pm 0.03$ & $17.49 \pm 0.05$ & $16.1 \pm 0.1$ \\
$\# 7$ & $20.15 \pm 0.05$ & $19.09 \pm 0.03$ & $17.33 \pm 0.05$ & $17.8 \pm 0.4$ & - \\
$\# 8$ & - & - & $17.88 \pm 0.09$ & $17.4 \pm 0.3$ & $15.6 \pm 0.1$ \\
$\# 9$ & - & - & $17.43 \pm 0.06$ & $15.97 \pm 0.09$ & $14.5 \pm 0.2$ \\
$\# 10$ & - & $20.48 \pm 0.09$ & $17.23 \pm 0.04$ & - & - \\
$\# 11$ & - & - & $18.11 \pm 0.07$ & $17.5 \pm 0.2$ & $16.0 \pm 0.1$ \\
$\# 12$ & - & $20.7 \pm 0.1$ & $17.84 \pm 0.07$ & $18.2 \pm 0.2$ & - \\
\hline$A M$ & 1.054 & 1.052 & 1.150 & 1.114 & 1.076 \\
$Z_{\mathrm{p}}$ & $-0.44 \pm 0.01$ & $0.39 \pm 0.01$ & $1.84 \pm 0.02$ & $2.01 \pm 0.02$ & $2.6 \pm 0.02$ \\
ext & 0.091 & 0.051 & $0.13 \pm 0.02$ & $0.10 \pm 0.02$ & $0.07 \pm 0.02$ \\
\hline
\end{tabular}

${ }^{\dagger}$ From Fig. $7 ; \stackrel{\ddagger}{\ddagger}$ located in the XMM-Newton error circle.

Note: At the bottom, we show the parameters for the photometric solutions: $A M$ the airmass, $Z_{\mathrm{p}}$ the zero-point and ext the extinction.

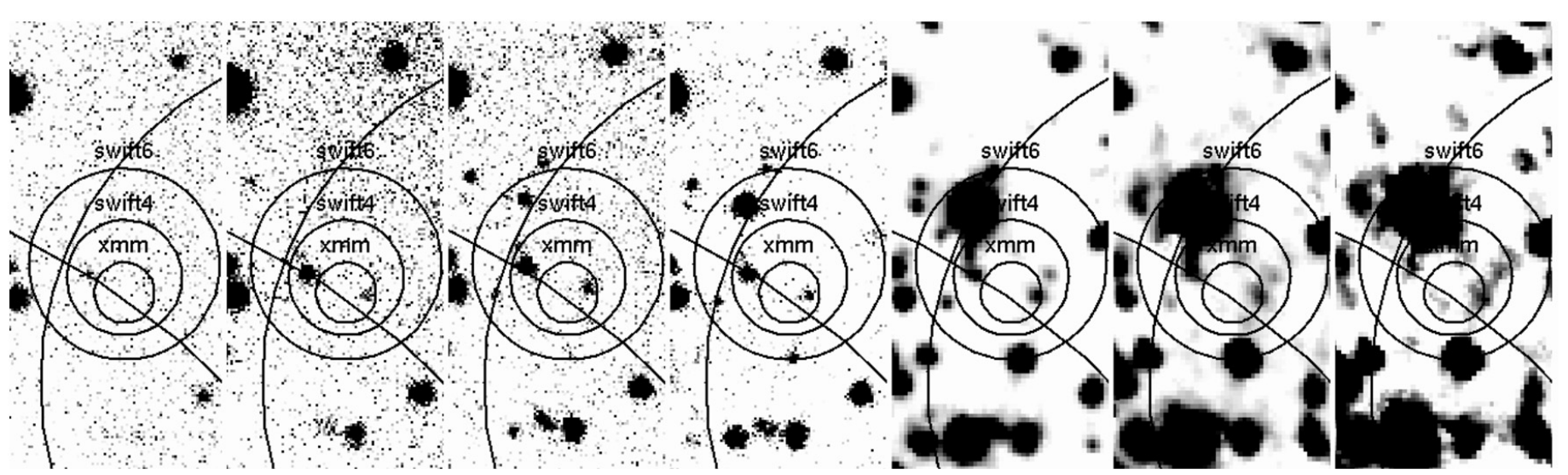

Fig. 8. Left to right: images in filters: $V, R, I, Z, J, H$, and $K_{\mathrm{s}}$. The only candidate counterpart is visible from filters $R$ to $K_{\mathrm{s}}$.

because of the detections in pointings 01100038 and 01730024 , where the rate varies by a factor 3-4 on a timescale of $1 \mathrm{~h}$ in comparison to the average rates during the outbursts. Still, these detections may also be due to a sensitivity bias, because the count rates are similar to the $5.1 \sigma$ detection threshold.

A spin-down of AX J1749.1-2733 is observed between the 2 observations of the bright flare with INTEGRAL and the $X M M$-Newton observation. We derived a spin-down of $\dot{P}=$ $0.08 \pm 0.02 \mathrm{~s} \mathrm{yr}^{-1}$ (i.e. $\left.\dot{v}=(-6.2 \pm 1.4) \times 10^{-13} \mathrm{~Hz} \mathrm{~s}^{-1}\right)$. Such spin-down episodes have already been observed in other accreting pulsars, some examples being EXO 2030+375 with $\dot{v}=$ $-3.4 \times 10^{-14} \mathrm{~Hz} \mathrm{~s}^{-1}$ and A0535+26 with $\dot{v}=-2.2 \times 10^{-13} \mathrm{~Hz} \mathrm{~s}^{-1}$ (Bildsten et al. 1997, and references therein). To have accretion onto an NS, the magnetic radius $R_{\text {mag }}$ must be lower than the co-rotation radius $R_{\text {cor }}$. Otherwise, the infalling matter is centrifugally expelled by the magnetic field (the propeller regime, Illarionov \& Sunyaev 1975). For an NS $\left(M=1.4 M_{\odot}, R=\right.$ $10 \mathrm{~km}$ ) with a spin period of $66 \mathrm{~s}$, at the equilibrium point $R_{\text {mag }}=R_{\text {cor }}$, one estimates the magnetic field to be $B=6.5 \times$ $10^{12} \mathrm{G} \times\left(\dot{M} / 10^{-10} M_{\odot} \mathrm{yr}^{-1}\right)^{1 / 2}$. The magnetic radius depends on the accreted matter flow as $R_{\text {mag }} \propto \dot{M}^{-2 / 7}$. Thus, the magnetic radius may exceed the co-rotation radius if the accretion rate becomes low enough, stopping the accretion. The infalling matter, expelled by the propeller effect, can then remove some angular momentum and decrease the spin period of the neutron star. With such a long orbital period, the NS is in fact usually far from the dense accreting zone, where such effects leading to a decrease in the spin period can occur.

The broad X- and hard X-ray spectrum of AX J1749.1-2733 is similar to other HMXB pulsars (White et al. 1995). It is well-fitted with an absorbed power law and a high-energy cutoff with values $N_{\mathrm{H}}=20.1_{-1.3}^{+1.5} \times 10^{22} \mathrm{~cm}^{-2}, \Gamma=1.0_{-0.3}^{+0.1}$, and $E_{\text {cut }}=21_{-3}^{+5} \mathrm{keV}$. The large absorption is in good agreement with the previous observation (ASCA, Swift; Sakano et al. 2002; Romano et al. 2007; Kong 2007); still, it is strongly constrained with the EPIC/pn data. This large absorption is an order of magnitude higher than the galactic absorption expected in the line of sight of AX J1749.1-2733, $N_{\mathrm{H}}(\mathrm{HI})=1.7 \times 10^{22} \mathrm{~cm}^{-2}$ (interpolated from the HI map of Dickey \& Lockman 1990). Thus, the $\mathrm{X}$-ray source is intrinsically absorbed. The combination of the EPIC/pn and IBIS/ISGRI spectra allowed us to better constrain the continuum getting a harder spectrum with a $\Gamma$ that decreases by a factor 2 in comparison with former estimates and the necessity of the high-energy cutoff.

Only one candidate counterpart is located within the most accurate X-ray position of XMM-Newton (see Sect. 3.1). Using the relation of Predehl \& Schmitt (1995) that links the X-ray absorption and the optical extinction, $N_{\mathrm{H}}\left(\mathrm{cm}^{-2}\right) / A_{\mathrm{V}}(\mathrm{mag})=$ $1.79 \times 10^{21}$, the expected optical extinction $A_{\mathrm{V}}$ is 9.5 mag assuming the reported galactic $N_{\mathrm{H}}=1.7 \times 10^{22} \mathrm{~cm}^{-2}$. Then, the NIR extinctions are estimated as $A_{J}=2.680, A_{H}=1.663$ and $A_{K}=1.064$ using $A_{J} / A_{\mathrm{V}}=0.282, A_{H} / A_{\mathrm{V}}=0.175$ and 


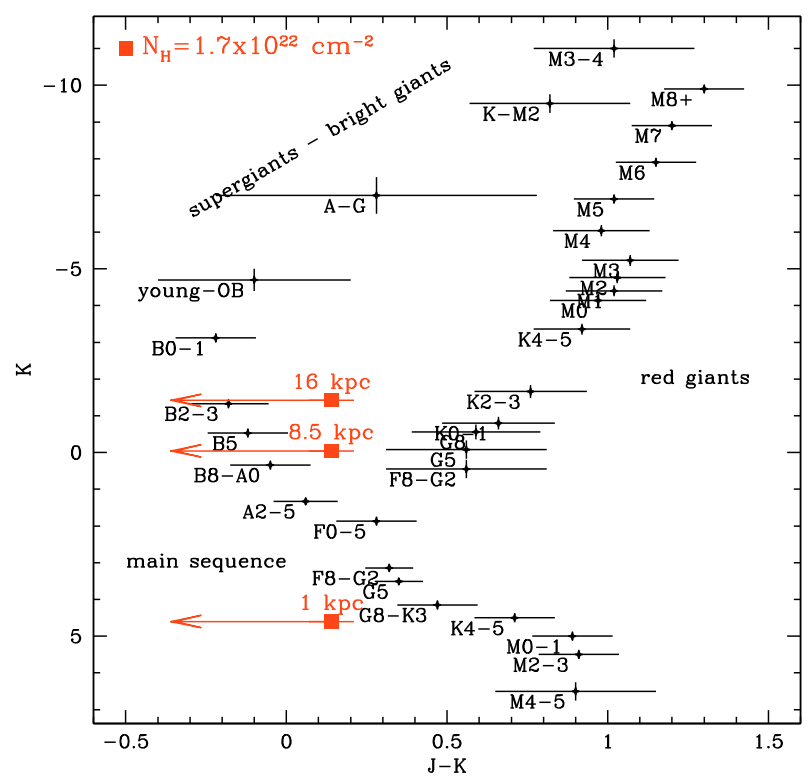

Fig. 9. Colour-magnitude diagram. The magnitudes here correspond to absolute magnitude. The location of the source \#5 in the diagram is shown for the galactic $N_{\mathrm{H}}$ and several distances (1, 8.5 and $16 \mathrm{kpc}$ ). The arrows simulate the colour shift of $J-K \leq-0.5$ due to a disc-like ring of matter surrounding the star, assuming that it is a Be star.

$A_{\mathrm{K}} / A_{\mathrm{V}}=0.112$ (Rieke \& Lebofsky 1985). We derive the absolute magnitude as $M_{\mathrm{abs}}=m_{\mathrm{app}}-5 \log ($ distance $/ 10 \mathrm{pc})-A_{\mathrm{V}}$. Assuming a distance of $8.5 \mathrm{kpc}$, we obtain $M_{J}=0.09, M_{H}=$ 0.39 and $M_{K}=-0.05^{13}$, so the colour is $(J-K)_{0}=0.14$. Thus, in a colour-magnitude diagram (CMD), the candidate companion star of AX J1749.1-2733 falls near the zone of the main sequence stars with spectral type between B5 and B8-A0 (see Fig. 9). In this case of a BeXB system, the disc-like ring of matter surrounding the B star can modify its colour $J-K$ by a large factor (e.g. up to $-0.5 \mathrm{mag}$ in $4 \mathrm{U} 0115+63$ Reig et al. 2007). This is illustrated in Fig. 9 by arrows of maximum length of $0.5 \mathrm{mag}$ that strengthen the scenario of a B star located far away in the Galaxy, thus highly absorbed by the interstellar medium. In another case where the star would be located nearer to us $(\sim 1 \mathrm{kpc})$, thus with a lower extinction, the star would be a main sequence star of type KM. In that case, this star would probably not be the counterpart of AX J1749.1-2733, because the large orbital period completely rules out the possibility of combining such KM star with this X-ray source. The possibility that the star is a red giant is also unlikely as it should combine a location far away in the Galaxy with low extinction. Spectroscopy of the candidate counterpart is needed to disentangle between the 2 possibilities, either a B star located far away in the Galaxy $\gtrsim 8.5 \mathrm{kpc}$ and strongly absorbed or a normal star located nearer to us. Still, because of the X-ray properties observed for AX J1749.1-2733 and the absence of any other candidate, the B star is favoured. As the known BeXB have an earlier spectral type than B3 (Negueruela 1998), this would imply that the source is located at a very long distance of $>8.5 \mathrm{kpc}$. Another argument in favour of the BeXB scenario is the reddening-free parameter $Q=(J-H)-1.70\left(H-K_{\mathrm{S}}\right)$ (Negueruela \& Schurch 2007). For candidate \#5, $Q \sim-0.9 \pm 0.1$, thus suggesting a high IR excess. Such excess is not expected in KM stars whose $Q$ values lie between $0.4-0.5$; however, a factor $Q<0$ is expected for

\footnotetext{
13 The $K_{\mathrm{s}}$ magnitude is transformed into the $K$ magnitude using the relation $K-K_{\mathrm{s}}=-0.005(J-K)$ (SOFI user manual, p. 7).
}

Be stars. Assuming a distance of $8.5 \mathrm{kpc}$, the $22-50 \mathrm{keV}$ luminosity is $3 \times 10^{36} \mathrm{erg} \mathrm{s}^{-1}$ during the bright flare that occurred on MJD 52891 and $0.4-0.9 \times 10^{36} \mathrm{erg} \mathrm{s}^{-1}$ during the long outbursts, values typical of BeXB. The $0.2-10 \mathrm{keV}$ luminosity is $0.2 \times 10^{36} \mathrm{erg} \mathrm{s}^{-1}$, a factor 2-4 lower than in INTEGRAL. We point out that, in the case of a BeXB with typical outbursts of the order of $\sim 10^{36} \mathrm{erg} \mathrm{s}^{-1}$, and if the system was located nearer to us ( $\lesssim 8 \mathrm{kpc})$, INTEGRAL would detect such a source with a $22-50 \mathrm{keV}$ count rate $\gtrsim 2$ counts $\mathrm{s}^{-1}$, which is not the case.

In the case of a BeXB, AX J1749.1-2733 shows a high intrinsic absorption of $N_{\mathrm{H}}=20.1_{-1.3}^{+1.5} \times 10^{22} \mathrm{~cm}^{-2}$ that is quite uncommon in other BeXB. Indeed, most of the BeXB have density columns closer to the galactic absorption with $N_{\mathrm{H}} \sim 1-$ $3 \times N_{\mathrm{H}}^{\mathrm{Gal}}$ (see Fig. 15 of Bodaghee et al. 2007). Only one BeXB exhibits such high absorption: 2S 1845-024. The properties of this source are very similar to AX J1749.1-2733 with $P_{\text {orb }}=242.18 \pm 0.01 \mathrm{~d}$, outburst duration of $\sim 13 \mathrm{~d}$, $P_{\text {spin }}=94.9 \mathrm{~s}$ (with episodes of spin-up and spin-down) and $N_{\mathrm{H}}=25 \pm 10 \times 10^{22} \mathrm{~cm}^{-2}$ (Koyama et al. 1990; Soffitta et al. 1998; Finger et al. 1999, and references therein). They classified 2S 1845-024 as a BeXB (located at $10 \mathrm{kpc}^{14}$ ) from its X-ray properties; however, no counterpart has been identified yet. Thus, AX J1749.1-2733 might be the first identified counterpart BeXB with high intrinsic absorption similar to the obscured SGXB revealed by INTEGRAL. Spectral properties of pulsars, either SGXB or BeXB, are very similar (see e.g. White et al. 1995). As IBIS/ISGRI is not affected by absorption, INTEGRAL allowed a large population of obscured SGXB to be revealed with $N_{\mathrm{H}}$ values of $\gtrsim 10^{23} \mathrm{~cm}^{-2}$. That such highly-absorbed BeXB have not been observed yet (Bodaghee et al. 2007) is perhaps not so surprising since BeXB are mostly transient sources, and the sources must also be observed during the outbursts by another X-ray emission with high sensitivity at low energies (such as XMM-Newton or Chandra) in order to constrain the absorption. Such a population of obscured BeXB might still have to be revealed. Indeed, the only BeXB among the INTEGRAL sources reported in Fig. 15 of Bodaghee et al. (2007) is the 3rd highly-absorbed BeXB (after AX J1749.1-2733 and 2S 1845-024).

All the X-ray properties observed in AX J1749.1-2733 lead to classifing this object as an X-ray binary, most probably an HMXB with a Be companion star whose compact object is an NS. The only optical/NIR candidate counterpart located inside the best X-ray error circle is compatible with a B star located far inside the galaxy and suffering large extinction. Besides, the lack of a supergiant companion and the duration/smoothness of the outbursts rule out the classification of this source as an SFXT, as proposed in in't Zand (2005) and Sguera et al. (2006). Instead, AX J1749.1-2733 seems to be an obscured BeXB located far away in the Galaxy.

Acknowledgements. The authors warmly thank the XMM-Newton team for accepting the ToO request. S.C. thanks the ESO staff, and especially Valenti Ivanov, for performing the ToO observations. We also warmly thank the referee I. Negueruela for his useful comments that improved the manuscript. We also thank Jérôme Rodriguez for a careful re-reading of the manuscript and for giving useful comments. We would like to thank A. Lutovinov for discussing the nature of this source at the 5th INTEGRAL Anniversary workshop.

14 This value can be considered as an upper limit as they derive it taking the minimum observed $N_{\mathrm{H}}$ as interstellar absorption without taking the intrinsic absorption into account. 


\section{References}

Arnaud, K. A. 1996, in Astronomical Data Analysis Software and Systems V, ed. G. H. Jacoby \& J. Barnes, ASP Conf. Ser., 101, 17

Bildsten, L., Chakrabarty, D., Chiu, J., et al. 1997, ApJS, 113, 367

Bird, A. J., Malizia, A., Bazzano, A., et al. 2007, ApJS, 170, 175

Bodaghee, A., Courvoisier, T. J.-L., Rodriguez, J., et al. 2007, A\&A, 467, 585

Coe, M. J. 2000, in The Be Phenomenon in Early-Type Stars, ed. M. A. Smith,

H. F. Henrichs, \& J. Fabregat, IAU Colloq. 175, ASP Conf. Ser., 214, 656

Corbet, R. H. D. 1984, A\&A, 141, 91

Corbet, R. H. D. 1986, MNRAS, 220, 1047

Courvoisier, T. J.-L., Walter, R., Beckmann, V., et al. 2003, A\&A, 411, L53

Dickey, J. M., \& Lockman, F. J. 1990, ARA\&A, 28, 215

Finger, M. H., Bildsten, L., Chakrabarty, D., et al. 1999, ApJ, 517, 449

Götz, D., Falanga, M., Senziani, F., et al. 2007, ApJ, 655, L101

Grebenev, S. A., \& Sunyaev, R. A. 2007, Astron. Lett., 33, 149

Gros, A., Goldwurm, A., Cadolle-Bel, M., et al. 2003, A\&A, 411, L179

Horne, J. H., \& Baliunas, S. L. 1986, ApJ, 302, 757

Illarionov, A. F., \& Sunyaev, R. A. 1975, A\&A, 39, 185

in't Zand, J. J. M. 2005, A\&A, 441, L1

Jansen, F., Lumb, D., Altieri, B., et al. 2001, A\&A, 365, L1

Karasev, D., Tsygankov, S., Lutovinov, A., Churazov, E., \& Sunyaev, R. 2007, The Astronomer's Telegram, 1245, 1

Karasev, D. I., Tsygankov, S. S., \& Lutovinov, A. A. 2008, MNRAS, L50

Kong, A. K. H. 2007, The Astronomer's Telegram, 1039, 1

Koyama, K., Kunieda, H., Takeuchi, Y., \& Tawara, Y. 1990, PASJ, 42, L59

Kuulkers, E., Shaw, S. E., Paizis, A., et al. 2007, A\&A, 466, 595

Landolt, A. U. 1992, AJ, 104, 340

Lebrun, F., Leray, J. P., Lavocat, P., et al. 2003, A\&A, 411, L141

Leyder, J.-C., Walter, R., Lazos, M., Masetti, N., \& Produit, N. 2007, A\&A, 465, L35

Liu, Q. Z., van Paradijs, J., \& van den Heuvel, E. P. J. 2000, A\&AS, 147, 25

Liu, Q. Z., van Paradijs, J., \& van den Heuvel, E. P. J. 2006, A\&A, 455, 1165
Negueruela, I. 1998, A\&A, 338, 505

Negueruela, I. 2007, in Massive Stars in Interactive Binaries, ed. N. St.-Louis, \& A. F. J. Moffat, ASP Conf. Ser., 367, 477

Negueruela, I., \& Schurch, M. P. E. 2007, A\&A, 461, 631

Negueruela, I., Smith, D. M., Reig, P., Chaty, S., \& Torrejón, J. M. 2006, in The X-ray Universe 2005, ed. A. Wilson, ESA SP-604, 165

Pellizza, L. J., Chaty, S., \& Negueruela, I. 2006, A\&A, 455, 653

Persson, S. E., Murphy, D. C., Krzeminski, W., Roth, M., \& Rieke, M. J. 1998, AJ, 116, 2475

Predehl, P., \& Schmitt, J. H. M. M. 1995, A\&A, 293, 889

Press, W. H., \& Rybicki, G. B. 1989, ApJ, 338, 277

Psaltis, D. 2006, Accreting neutron stars and black holes: a decade of discoveries, Compact stellar X-ray sources, 1

Reig, P., Belloni, T., Israel, G. L., et al. 2008, ArXiv e-prints, 804

Reig, P., Larionov, V., Negueruela, I., Arkharov, A. A., \& Kudryavtseva, N. A. 2007, A\&A, 462, 1081

Rieke, G. H., \& Lebofsky, M. J. 1985, ApJ, 288, 618

Romano, P., Mangano, V., Cucchiara, A., \& Sidoli, L. 2007, The Astronomer's Telegram, 1040, 1

Sakano, M., Koyama, K., Murakami, H., Maeda, Y., \& Yamauchi, S. 2002, ApJS, 138,19

Sguera, V., Bazzano, A., Bird, A. J., et al. 2006, ApJ, 646, 452

Smith, D. M., Heindl, W. A., Markwardt, C. B., et al. 2006, ApJ, 638, 974

Soffitta, P., Tomsick, J. A., Harmon, B. A., et al. 1998, ApJ, 494, L203

Strüder, L., Briel, U., Dennerl, K., et al. 2001, A\&A, 365, L18

Turner, M. J. L., Abbey, A., Arnaud, M., et al. 2001, A\&A, 365, L27

Ubertini, P., Lebrun, F., Di Cocco, G., et al. 2003, A\&A, 411, L131

White, N. E., Nagase, F., \& Parmar, A. N. 1995, X-ray binaries, Cambridge Astrophysics Series (Cambridge, MA: Cambridge University Press), ed. W.H.G. Lewin, J. van Paradijs, E.J.P. Van den Heuvel, 1

Winkler, C., Courvoisier, T. J.-L., Di Cocco, G., et al. 2003, A\&A, 411, L1

Zurita Heras, J. A., Chaty, S., \& Rodriguez, J. 2007, The Astronomer's Telegram, 1035,1 